\title{
Color Detection and Mixing System
}

\author{
Khalid Ammar \\ Department of Computer Eng \\ Ajman University
}

Ajman, UAE

\author{
Amira Duhair \\ Department of Computer Eng \\ Ajman University \\ Ajman, UAE
}

\author{
Lara Bilal \\ Department of Computer Eng \\ Ajman University \\ Ajman, UAE
}

\begin{abstract}
The detection of the color value and the automation of its production is very useful for many industrial applications. [3, 4]. We have developed a microcontroller-based system that detects colors and controls the mixing of the primary colors to get the matching colors. The system can be used as a tool to determine the compositions of chosen colors and produce a matching colors that meets the requirement of the required colors. The system can make the production of the colors much faster, more reliable and more consistent. This document describes the design, the implementation of the system. The system can be used as a tool in different industries such as digital printing, textile, cosmetics, wallpaper, gift-wrap, building materials, signage, etc [3, 4].
\end{abstract}

Keywords: Digital paint, Color making and mixing, digital printing, paint products.

\section{INTRODUCTION}

All colors that we can see can be composed from a set of primary colors [5,6,7]. Detection of the composition of chosen color and its automated generation has a wide range of applications [3,4]. A color models (known as color spaces) can represent a chosen color using a set of primary colors mapped to the color model in discrete values. Many color models are used to describe colors, each model is used for different purposes and different areas of applications. We are going to focus on two of the most normally used color models, the RGB model and the CMYK model $[6,7]$. The two models are shown in shown in figure 1 .

The RGB color model is composed from three different colors, red, green and blue, this model used for sensing, and display in electronic systems, such as televisions and computer monitor.

The CMYK color model is composed from four different colors, cyan, magenta, yellow, and key (black), this model most commonly used for professional color printing. To produce any degree of color on the colors scale, our system uses five primary colors (the four CMYK colors and the fifth color is a white color $(\mathrm{W})$, the white color is used to adjust the brightness of the color.

\section{RGB / CMYK Color Space Conversion:}

RGB color has to be converted to CMYK color format before they are sent for mixing. In this work, the following formulas are used to convert the RGB color space to CMYK color space $[1,2]$.

$$
\begin{aligned}
& “ R^{`}=R / 255 \\
& G^{`}=G / 255 \\
& B^{`}=B / 255 \\
& K=1-\max \left(R^{\prime}, G^{\prime}, B^{\prime}\right) \\
& C=\left(1-R^{\prime}-K\right) /(1-K) \\
& M=\left(1-G^{\prime}-K\right) /(1-K) \\
& Y=\left(1-B^{\prime}-K\right) /(1-K)
\end{aligned}
$$

$C \max =\max \left(R^{\prime}, G^{\prime}, B^{\prime}\right)$

$C \min =\min \left(R^{\prime}, G^{\prime}, B^{\prime}\right)$

$\Delta=C \max -C \min$

Lightness calculation:

$L=(\operatorname{Cmax}+C \min ) / 2 \cdots$
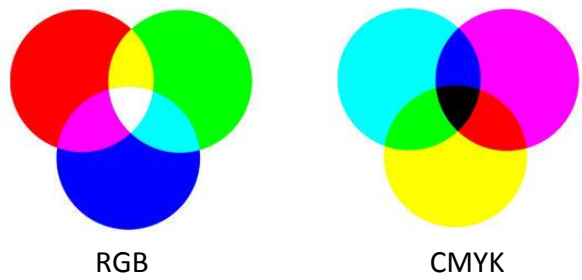

Figure. 1 The RGB and the CMYK Color models.

\section{The System Description}

The color Mixing System has been designed and implemented as a result of integrating various hardware and software technologies. Figure. 2 shows the overall block diagram of the system, the diagram illustrates how the system interact with each other. Figure 2 shows a general flow chart of the system software, the control program for the color mixing system is integrated in the controller, the program control the mixing of CMYK and white colors.

The system consists of a color sensor, a microcontroller circuit board, a GSM shield, a peristaltic pumps, a scale sensor, a primary color containers, and a mobile phone $[8,9$, $10,11]$. Figure 3 shows a general flow chart of the system software, which control the system.

The system detects the RGB value of the chosen color and compose the required color by mixing the primary color in the right proportions to get the exact match of the color. The color sensor determines the RGB value of the chosen color, also the RGB value of the chosen color can be determined by mobile phone using the mobile phone camera. In addition, the mobile application allows the user to enter the RGB value of the chosen color manually. We have used android mobile smartphone to implement this application. 


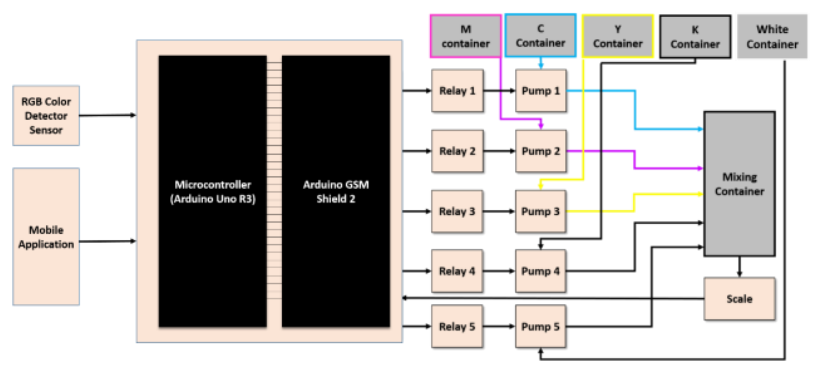

Figure. 2 The system functional block diagram.

The color sensor is connected to the microcontroller and its RGB reading is directly conveyed to the Microcontroller, the mobile application sends the RGB values to the microcontroller via GSM shield. The microcontroller convers the received RGB value to CMYK values. In addition, the microcontroller determines the amount of the CMYK and white colors which will be mixed together to get the exact color.

The system uses five tanks of primary colors to generate the chosen color, the primary colors are, cyan, magenta, yellow, black and white $\mathrm{W}$ colors. Each tank has independent peristaltic pump connected to its outlet pipe. Under the supervision of the microcontroller, the pumps gets the appropriate amount of color from the appropriate thank and dispense it into the mixing container.

A scale sensor is used to senses the quantity of paint inside the mixing container and gives a feedback to the microcontroller about the precise amount inside the mixing container; this is to get the exact quantity of the chosen paint. The delivery of the primary colors to the mixing container stops once it reached the right quantity.

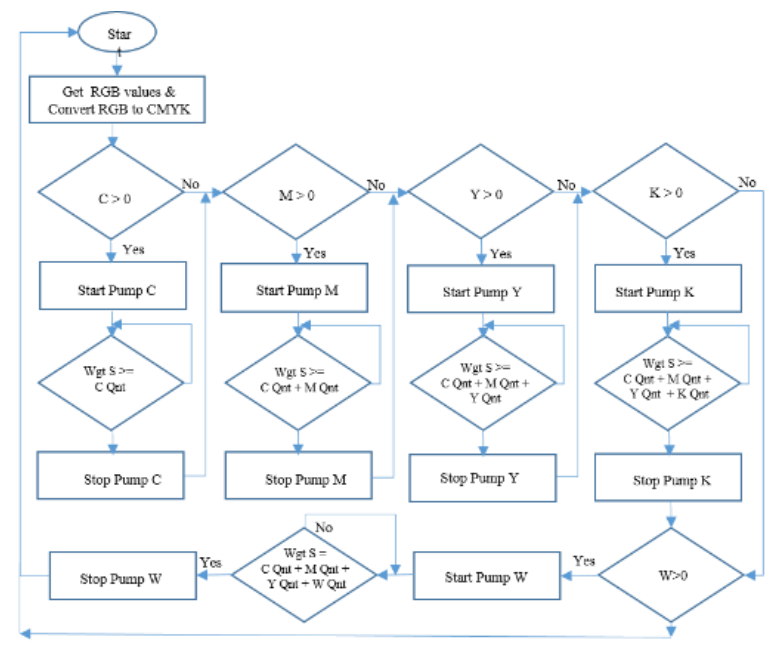

Figure.3 The system general flow chart.

The microcontroller software implements the equations that converts the RGB color model to the CMYK color model and controls the mixing of the required color. We have used $\mathrm{C}$ Programming to implement these equations. The general flow chart of the system software is shown in figure 3 . Figure 4 shows the general flow chart of the mobile application.

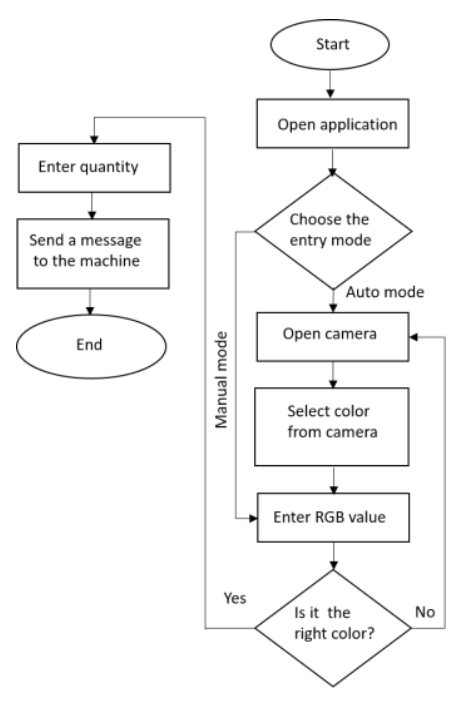

Figure.4 Application flow chart.

Figure 5 shows the layout of the Smartphone application. We have used android studio in the development of the mobile application. In the first page of the application shown in figure $5 \mathrm{a}$, a color wheel icon takes the user to the mode selection page shown in figure $5 \mathrm{~b}$, where users can select the entry mode; users can select either the auto mode selection or the manual mode selection.
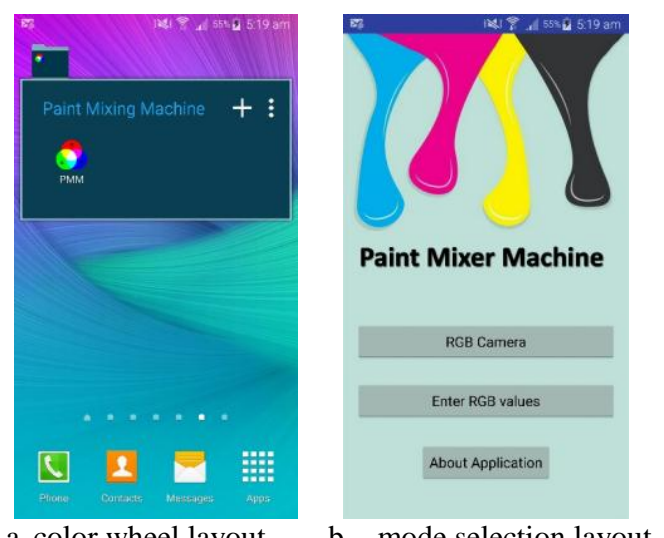

a. color wheel layout

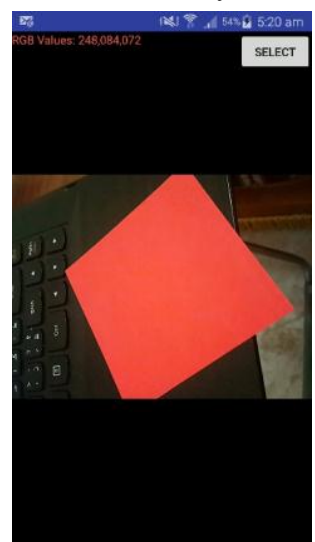

b. mode selection layout

c. Auto mode layout

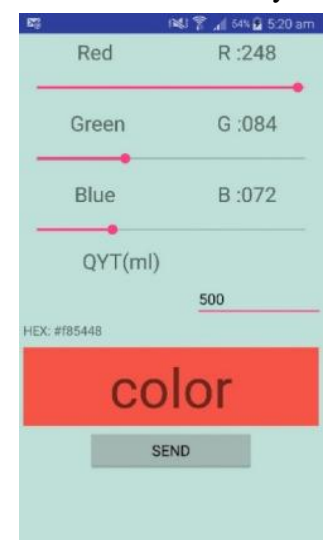

d. Manual mode layout

Figure.5 The layout of the Smartphone Application

- In the auto mode, the chosen color can be selected through the mobile's camera shown in figure $5 \mathrm{c}$. The RGB values of the chosen color is determined by tapping on the screen on the area of that color. 
- In the manual mode, the RGB value of the chosen color is selected manually through the manual mode layout page shown in figure $5 \mathrm{~d}$. Three seek bars allows the user to choose the RGB values (from 0 to 255). After capturing the RGB values, the user can choose the quantity of the chosen paint color, and then the user can send the values of the RGB color and the desired quantity of that color to the microcontroller for processing.

\section{Tests and Results}

Several tests have been carried out for evaluating the validity of the system and the correctness of the color match. The system detected the colors under test successfully and an appropriate colors match were generated appropriately. Figure 6a shows the detection of a blue color through the RGB sensor. Figure $6 \mathrm{~b}$ shows the creation of the color match.

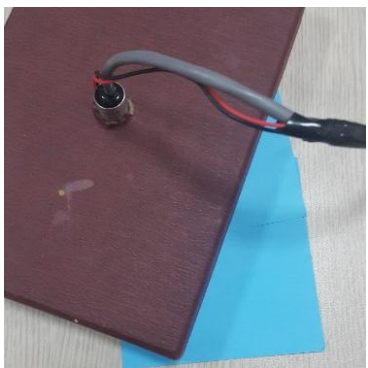

a. Blue color detection.

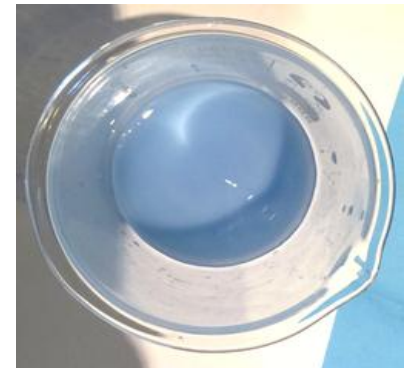

b. Blue color Produced
Figure.6 Color test through the RGB sensor.

Figure 7a shows the detection of a red color through the mobile camera. Figure $7 \mathrm{~b}$ shows the creation of the chosen color match.

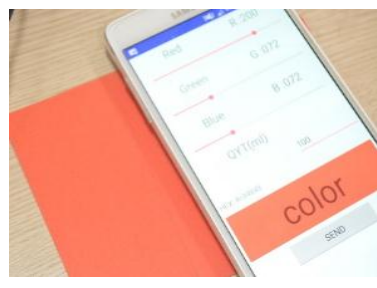

a. Detection of the Red color

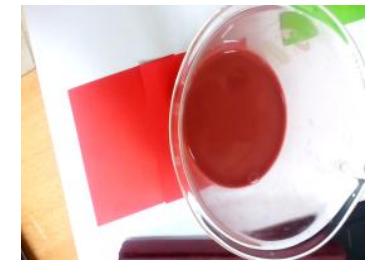

b. The Red color Produced
Figure.7 Color test through the mobile camera.

Figure 8a show the manual entry of the yellow color. Figure $8 \mathrm{~b}$ shows the creation of the chosen color match.

\section{System capability}

The developed system is capable of generating a various collection of colors. User can reproduce chosen colors and quantity using the onboard RGB color sensor, or remotely can use his/her mobile camera. User can set his own color ratio manually to make a range of colors. The system can be used as a tool in different industries such as digital printing, textile, cosmetics, wallpaper, gift-wrap, building materials, etc. [3, 4].
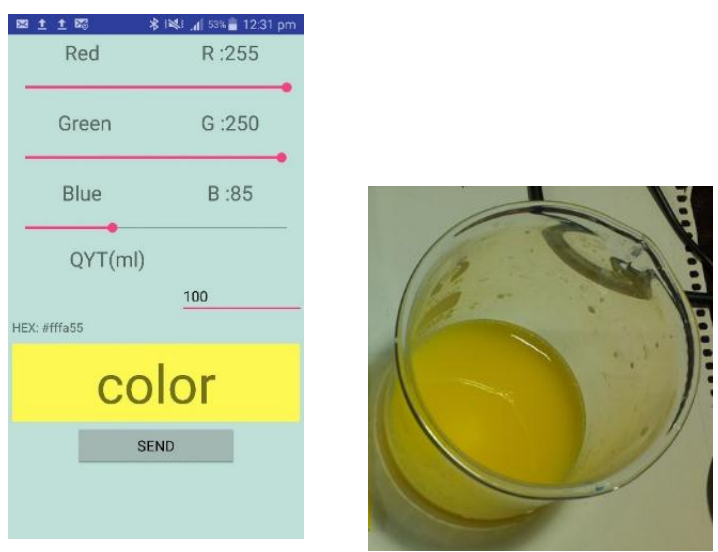

a. Yellow color detection. b. Yellow color Produced

Figure.8 Color test through manual entry.

\section{Conclusion}

In this paper we have described the design and the implementation of the color detection and mixing system. All the design goals were brought into realization. The system has clearly demonstrated that it's capable of producing the matching color in the color spectrum. In addition to the manual RGB entry, two different implementations have been designed and tested, the first implementation used the mobile camera and second implementation used the RGB sensor. The system can be used as indispensable tool for various industrial application such as digital printing, textile, cosmetics, building materials, etc.

\section{References}

[1] https://www.vocal.com/video/rgb-and-cmyk-color-spaceconversion/

[2] http://www.rapidtables.com/convert/color/rgb-to-hsl.htm

[3] https://www.spoonflower.com/welcome

[4] http://www.smitherspira.com/news/2015/july/printedsignage-market-set-to-reach-\$49-billion

[5] https://en.wikipedia.org/wiki/RGB_color_model

[6] https://en.wikipedia.org/wiki/CMYK_color_model

[7] http://www.rapidtables.com/convert/color/rgb-to-hsl.htm

[8] https://www.arduino.cc/en/Main/ArduinoBoardUno

[9] https://www.arduino.cc/en/Main/ArduinoGSMShield

[10] https://www.atlasscientific.com/_files/_datasheets/_probe/ENV-RGB.pdf

[11] https://en.wikipedia.org/wiki/Peristaltic_pump 